\title{
The effect of intermittent energy and carbohydrate restriction $v$. daily energy restriction on weight loss and metabolic disease risk markers in overweight women
}

\author{
Michelle Harvie $^{1 *}$, Claire Wright ${ }^{2}$, Mary Pegington ${ }^{1}$, Debbie McMullan ${ }^{1}$, Ellen Mitchell ${ }^{1}$, \\ Bronwen Martin ${ }^{3}$, Roy G. Cutler ${ }^{4}$, Gareth Evans ${ }^{1}$, Sigrid Whiteside ${ }^{5}$, Stuart Maudsley ${ }^{4}$, \\ Simonetta Camandola ${ }^{3}$, Rui Wang ${ }^{3}$, Olga D. Carlson ${ }^{3}$, Josephine M. Egan ${ }^{3}$, \\ Mark P. Mattson ${ }^{4}$ and Anthony Howell ${ }^{1}$ \\ ${ }^{1}$ Genesis Breast Cancer Prevention Centre, University Hospital of South Manchester NHS Foundation Trust, \\ Southmoor Road, Manchester M23 9LT, UK \\ ${ }^{2}$ Department of Clinical Sciences and Nutrition, University of Chester, Cheshire, UK \\ ${ }^{3}$ Laboratory of Clinical Investigation, National Institute on Aging Intramural Research Program, Baltimore, MD, USA \\ ${ }^{4}$ Laboratory of Neurosciences, National Institute on Aging Intramural Research Program, Baltimore, MD, USA \\ ${ }^{5}$ Department of Statistics, University Hospital of South Manchester NHS Foundation Trust, Southmoor Road, \\ Manchester M23 9LT, UK
}

(Submitted 7 September 2012 - Final revision received 13 February 2013 - Accepted 13 February 2013 - First published online 16 April 2013)

\begin{abstract}
Intermittent energy restriction may result in greater improvements in insulin sensitivity and weight control than daily energy restriction (DER). We tested two intermittent energy and carbohydrate restriction (IECR) regimens, including one which allowed ad libitum protein and fat (IECR + PF). Overweight women $(n 115)$ aged 20 and 69 years with a family history of breast cancer were randomised to an overall $25 \%$ energy restriction, either as an IECR $(2500-2717 \mathrm{~kJ} / \mathrm{d},<40 \mathrm{~g}$ carbohydrate/d for $2 \mathrm{~d} /$ week) or a $25 \%$ DER (approximately $6000 \mathrm{~kJ} / \mathrm{d}$ for $7 \mathrm{~d}$ /week) or an IECR + PF for a 3-month weight-loss period and 1 month of weight maintenance (IECR or IECR + PF for $1 \mathrm{~d} /$ week). Insulin resistance reduced with the IECR diets (mean - 0.34 (95\% CI - 0.66, - 0.02) units) and the IECR + PF diet (mean - 0.38 (95\% CI $-0 \cdot 75,-0 \cdot 01)$ units). Reductions with the IECR diets were significantly greater compared with the DER diet (mean $0 \cdot 2$ (95\% CI $-0 \cdot 19$, $0.66) \mu \mathrm{U} /$ unit, $P=0.02$ ). Both IECR groups had greater reductions in body fat compared with the DER group (IECR: mean -3.7 ( $95 \%$ CI $-2.5,-4.9) \mathrm{kg}, P=0.007$; IECR + PF: mean $-3.7(95 \%$ CI $-2.8,-4.7) \mathrm{kg}, P=0.019$; DER: mean -2.0 (95\% CI $-1.0,3.0) \mathrm{kg})$. During the weight maintenance phase, $1 \mathrm{~d}$ of IECR or IECR + PF per week maintained the reductions in insulin resistance and weight. In the short term, IECR is superior to DER with respect to improved insulin sensitivity and body fat reduction. Longer-term studies into the safety and effectiveness of IECR diets are warranted.
\end{abstract}

Key words: Intermittent energy restriction: Low-carbohydrate diets: Weight loss: Daily energy restriction: Insulin resistance

The global health burden of obesity-related conditions such as diabetes, CVD, dementia and certain cancers, including breast cancer, may be reduced by weight loss and the associated improvements in insulin sensitivity. The difficulties of achieving and sustaining weight loss by energy restriction are well known ${ }^{(1)}$. Even when reduced weights are maintained, metabolic benefits achieved with weight loss are often attenuated because of non-compliance or adaptation ${ }^{(2-4)}$. Effective dietary interventions are needed that promote long-term adherence and sustained beneficial effects on metabolic and disease markers. Such interventions need to be palatable and satiating, meet minimal nutritional requirements, promote loss of fat and preserve lean body mass, ensure long-term safety, be simple to administer and monitor and have widespread public health utility.

Multiple dietary approaches have been studied that vary in macronutrient composition ${ }^{(5)}$ and the degree of energy restriction $^{(6)}$. These typically achieve long-term 5\% weight loss in

Abbreviations: DER, daily energy restriction; FFM, fat-free mass; HbA1c, glycated Hb; HOMA, homeostasis model assessment; IECR, intermittent energy and carbohydrate restriction; IECR + PF, intermittent energy and carbohydrate restriction + ad libitum protein and fat; IER, intermittent energy restriction; IGF-1, insulin-like growth factor 1; LOCF, last observation carried forward.

*Corresponding author: Dr M. Harvie, fax +44 161291 4421, email michelle.harvie@manchester.ac.uk 
$30-35 \%$ of subjects ${ }^{(7-9)}$. Recent large-scale, long-term (2-year), randomised studies have reported no significant differences in long-term compliance, weight loss ${ }^{(5)}$ or clinical effectiveness $^{(10)}$ between diets providing $15-65 \%$ of energy as carbohydrate and $25-40 \%$ of energy as protein. However, in shorter-term (3-4-month) studies, higher-protein diets providing 20-25\% energy as protein consistently report greater satiety, preferential loss of fat and preservation of fat-free mass (FFM) compared with lower-protein diets (15\% energy as protein) ${ }^{(11)}$.

Most weight-control programmes use daily energy restriction (DER), but intermittent energy restriction (IER) has been suggested as a possible alternative approach. IER may be easier to follow and potentially has greater positive metabolic effects since it includes repeated spells of more profound energy restriction than achieved with DER, albeit for short periods $^{(12)}$. In animal models, IER is superior or equivalent to DER with respect to longevity ${ }^{(13)}$, the reduction of breast ${ }^{(14)}$, prostate $^{(15)}$ and pancreatic ${ }^{(16)}$ cancers, and the reduction of cardiovascular and cerebrovascular disease ${ }^{(17)}$ and dementia ${ }^{(18)}$.

The optimal duration of restricted dietary periods within IER for weight loss and health benefits in human subjects is not known. Relatively short periods of restriction of up to $20 \mathrm{~h}$ appear to be ineffective ${ }^{(19)}$. Alternate-day fasting is feasible in human subjects but has not been directly compared with $\mathrm{DER}^{(20)}$. Also, three weekly periods of restriction reduce tumours in rodents but are unlikely to be acceptable in human subjects $^{(14)}$. Preliminary studies in human subjects have shown that periods of $2-7 \mathrm{~d}$ of IER may be as effective as DER for weight loss ${ }^{(21,22)}$, and may be linked to greater improvements in insulin sensitivity ${ }^{(22)}$ and lipid profiles ${ }^{(23)}$ compared with DER.

Here, we report the effectiveness with respect to the change in insulin resistance, weight and adiposity, of an intermittent energy and carbohydrate restriction (IECR, 2500-2717 kJ/d, $<40 \mathrm{~g} / \mathrm{d}$ of carbohydrate) for $2 \mathrm{~d} /$ week. Restricted days were low in carbohydrate and relatively high in protein and fat (20\% energy from carbohydrate, $45 \%$ protein and $35 \%$ fat), to maximise satiety and food choice and to limit the loss of FFM within the energy restriction period ${ }^{(11)}$. In an attempt to make the $2 \mathrm{~d}$ /week IECR diet more acceptable and further limit hunger and the loss of FFM, we also tested an alternative IECR diet that included unrestricted ('ad libitum') protein and fat (MUFA and PUFA) (IECR + PF), which was anticipated to self-limit energy intake to approximately $5000 \mathrm{~kJ} / \mathrm{d}$ (approximately 15\% energy as carbohydrate, 35\% protein and $50 \%$ fat) on restricted days. We compared the two low-carbohydrate IECR regimens to the widely used DER that is relatively high in protein with moderate fat and carbohydrate (45-50\% energy as carbohydrate, $20-25 \%$ protein and $30 \%$ fat) over 4 months including 3 months of weight loss and 1 month of weight-loss maintenance ${ }^{(11)}$.

\section{Methods}

\section{Study design and participants}

The present randomised trial was performed as a single-centre study at the Family History (Genetics) Clinic at the Genesis
Prevention Centre, University Hospital of South Manchester NHS Foundation Trust. Participants were enrolled between September 2009 and January 2011. Women were eligible for the study if their BMI was $24-45 \mathrm{~kg} / \mathrm{m}^{2}$ and/or body fat was $>30 \%$ of total weight, and their reported adult weight gain since the age of 20 years exceeded $7 \mathrm{~kg}$. There was no age restriction but subjects entered were between 20 and 69 years of age. Women were excluded if they were currently dieting or losing weight, or suffering from diabetes, major CVD, respiratory, psychiatric or musculoskeletal morbidity. All participants were at an increased risk of breast cancer by virtue of a family history (lifetime risk of breast cancer $\geq 16 \%$ assessed using the Tyrer-Cuzick risk prediction model $^{(24)}$ ), but had no personal history of breast cancer and had a normal mammogram within the previous 12 months. Women were approached by mail shot. Interested participants were screened to assess their physical and psychological health and motivation to lose weight by the study dietitians (M. H., C. W. and M. P.), and were asked to complete a trial of the $2 \mathrm{~d}$ IECR before recruitment. Of 142 eligible women identified after screening, eighteen women (12\%) were unable to tolerate the $2 \mathrm{~d}$ trial and a further nine $(6 \%) \mathrm{did}$ not try the diet, but felt they would not be able to follow the IECR diet for 4 months if allocated (Fig. 1). The study was conducted according to the guidelines laid down in the Declaration of Helsinki and the principles of Good Clinical Practice. All procedures involving human subjects were approved by the North Manchester Research Ethics Committee (reference 09/H1006/34). Written informed consent was obtained from all subjects. The trial registration number is ISRCTN52913838 (http://www.controlled-trials.com/ISRCTN5 2913838/).

\section{Randomisation and masking}

Eligible women were randomly assigned to the three dietary interventions 1:1:1 for a 3-month weight-loss period and a further 1-month period of weight stabilisation. Participants were stratified by baseline BMI (above or below the predicted median value of $27 \mathrm{~kg} / \mathrm{m}^{2}$ ) and menopausal status with postmenopausal defined as 12 months after the last menstrual period or a surgically induced menopause. Group allocation was established by opaque, sealed envelopes that contained the assignment for each subject. Personnel performing laboratory measurements, and inputting and analysing trial data were blinded to group allocations. Anthropometric measures were performed by research dietitians who were not blinded to the treatment groups.

\section{Dietary interventions}

The IECR and DER interventions involved an overall 25\% energy restriction. This was calculated from estimated baseline energy requirements using reported metabolic equivalent of task $\times$ estimated $\mathrm{BMR}^{(25,26)}$ and ranged from 5040 to $7560 \mathrm{~kJ} / \mathrm{d}$ among the participants. The DER group was prescribed a daily energy-restricted Mediterranean-type diet that was relatively high in protein (25\% energy) with moderate

study at the Family Histry (Genetics) Clinic at the Genesis 


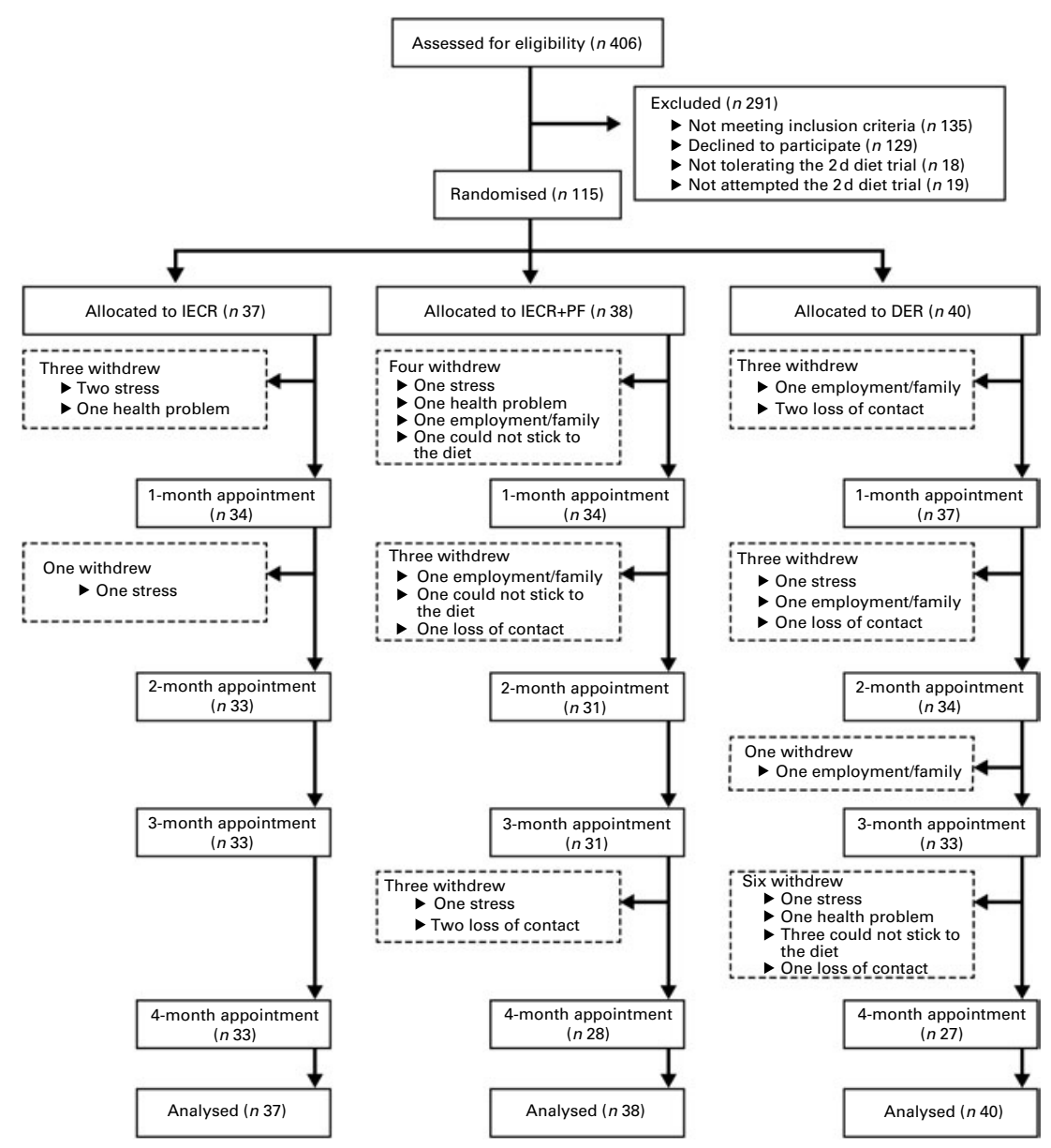

Fig. 1. CONSORT (Consolidated Standards of Reporting Trials) screening, recruitment and withdrawal information. IECR, intermittent energy and carbohydrate restriction; IECR + PF, IECR + ad libitum protein and fat; DER, daily energy restriction.

carbohydrate ( $45 \%$ energy from low-glycaemic-index carbohydrates) and moderate fat (30\% fat; $15 \%$ MUFA, $8 \%$ PUFA and $7 \%$ SFA) intakes ${ }^{(27)}$. It included five fruit and vegetable portions, nuts and seeds, whole-grain cereals, olive oil, fish and seafood, a moderate consumption of dairy products, poultry, eggs, and lean red meat and processed meat $(<400 \mathrm{~g} / \text { week })^{(28)}$.

Participants randomised to IECR were asked to restrict energy and carbohydrate on two consecutive days each week ( $70 \%$ energy restriction and $40 \mathrm{~g}$ carbohydrate) and to consume a euenergetic Mediterranean-type diet that met their estimated energy requirements for the remaining $5 \mathrm{~d}$ of the week. Restricted IECR days provided between 2500 and $2717 \mathrm{~kJ}$ and included approximately $250 \mathrm{~g}$ of protein foods including lean meat, fish, eggs, tofu, textured vegetable protein, three servings of low-fat dairy foods (e.g. $195 \mathrm{ml} \mathrm{semi}$ skimmed milk, $150 \mathrm{~g}$ low-fat yoghurt, $30 \mathrm{~g}$ low-fat cheese), four portions of low-carbohydrate vegetables and one portion of low-carbohydrate fruit, at least $1170 \mathrm{ml}$ of other low-energy fluids, and an over-the-counter multivitamin and mineral supplement providing the RDA for vitamins and typically $20-50 \%$ for minerals on restricted days. Participants randomised to IECR + PF had a diet virtually identical to the IECR group but were allowed unlimited lean meat, fish, eggs, tofu,
MUFA and PUFA on restricted days. Both intermittent diets were limited in SFA $(<10 \%$ energy). Participants in all the three intervention groups were advised to limit but not necessarily abstain from alcohol.

At 3 months, women were advised to follow euenergetic diets for weight maintenance based on revised requirement estimates using weight and activity levels measured at this time. The DER group continued with a daily euenergetic Mediterranean-type diet, while the IECR and IECR + PF groups included one restricted day per week and the standard Mediterranean-type diet for the remaining $6 \mathrm{~d}$.

The diets were not provided centrally, but were selfselected using detailed individualised food portion lists, meal plans and recipes. All participants were advised to gradually increase the frequency and intensity of exercise according to current activity levels, to aim to undertake $5 \times 45 \mathrm{~min}$ of moderate activity/week (metabolic equivalent of task $>3$ ). Each participant received a booklet outlining a home-based programme of walking, strengthening, toning and flexibility exercises $^{(29)}$. Diet and exercise advice was provided and reinforced for each subject by their designated study dietitian. Study dietitians used a range of accepted behaviour change techniques that included discussing the consequences of behaviour change in relation to the risk of breast cancer and 
other diseases ${ }^{(30)}$. Women received fortnightly motivational phone calls and monthly clinic appointments where they received feedback on their progress with weight and anthropometric measurements, and the revision of diet and exercise behavioural goals. All participants were encouraged to use a range of established cognitive behavioural techniques to adhere to their diet and exercise ${ }^{(30)}$.

\section{Study measurements}

Measurements were made before starting each diet and after 1, 3 and 4 months. Measurements were weight, total body fat and FFM determined by multi-frequency bioelectrical impedance (MC180MA; Tanita Europe BV), waist, hip and bust circumferences, systolic and diastolic blood pressure (BP Dinamap Carescape V100; GE Healthcare Technologies) and blood and urine sampling. All assessments were conducted in the morning after a $12 \mathrm{~h}$ fast. Subjects were asked to abstain from vigorous activity and alcohol for $24 \mathrm{~h}$ before the assessment. They were asked to drink water on the assessment morning and emptied their bladder immediately before the weight and impedance measurements. Both IECR groups were assessed at least $5 \mathrm{~d}$ after their weekly $2 \mathrm{~d}$ restriction to avoid any potential acute effects of the $2 \mathrm{~d}$ restriction on weight or blood markers which we reported previously ${ }^{(22)}$. Weight and body fat were assessed wearing light clothing. Body circumferences were measured in triplicate; waist was measured at the umbilicus and hips at the widest point over the trochanters. Blood pressure was measured in triplicate after $10 \mathrm{~min}$ at rest.

Fasting insulin, glucose, cholesterol, TAG, HDL-cholesterol (analysed as described previously ${ }^{(22)}$ ) and glycated $\mathrm{Hb}$ (HbA1c, ion-exchange HPLC method, Bio-Rad turbo HbA1c, Bio-Rad Laboratories Limited, CV 1.3\%) were measured at the Clinical Biochemistry Department at the University Hospital of South Manchester NHS Foundation Trust ${ }^{(22)}$. Fasting insulin and glucose were combined to calculate the insulin resistance index using the homeostasis model assessment (HOMA $)^{(31)}$. LDL-cholesterol was calculated using the formula of Friedewald et al. ${ }^{(32)}$. The remaining assays were performed at the National Institute on Ageing. Plasma leptin and adiponectin, plasma and urine ketone bodies were analysed as described previously ${ }^{(22)}$, and plasma and urine oxidative stress markers (advanced oxidation protein products) analysed using a modified method of Selmeci et al. ${ }^{(33)}$. Serum insulin-like growth factor 1 (IGF-1, CV 5.9\%), IL-6 (CV 2.9\%) and TNF- $\alpha$ (CV 4.9\%) were measured using ELISA from Alpco Diagnostics, R\&D Systems and Linco-Millipore, respectively. The leptin:adiponectin ratio was also estimated ${ }^{(34,35)}$. All blood and urine samples were stored at $4^{\circ} \mathrm{C}$ and frozen at $-70^{\circ} \mathrm{C}$ within $24 \mathrm{~h}$. Blood samples were centrifuged within $4 \mathrm{~h}$ of collection to separate the serum from the cells. Samples were batched so that all samples from a participant were included in the same assay ${ }^{(36)}$. After 3 months on the diets, a subset of women (six IECR and five IECR + PF) provided fasting samples on the morning immediately before and the morning immediately after their $2 \mathrm{~d}$ restriction, and then after $2 \mathrm{~d}$ of their unrestricted Mediterranean diet to assess any acute effects of the diets on disease risk markers.

Adherence to the dietary interventions at 1, 3 and 4 months was assessed using $7 \mathrm{~d}$ food diaries checked for completeness with the respondent. Mean daily energy, protein, carbohydrate, total fat, SFA, PUFA, MUFA, fibre (Association of Official Analytical Chemists method) and weekly alcohol intakes were estimated using WISP (Tinuviel Software). In addition, both IECR groups were asked to record whether they had successfully completed either 0,1 or $2 \mathrm{~d}$ of restriction each week during the study period.

Hunger and appetite were assessed using validated visual analogue scales ${ }^{(37)}$ at the end of the first and second restricted days and after five unrestricted days, and for three corresponding days of the week for the DER group. Physical activity was assessed from $7 \mathrm{~d}$ activity diaries, which recorded specific activities and sedentary behaviours and categorised the intensity of activity using a ten-point effort rating scale $^{(38)}$. Trained investigators blinded to the group allocation categorised activities into metabolic equivalents of energy and hence the time spent in moderate (metabolic equivalent of task 3-5.9) or vigorous (metabolic equivalent of task $>6.0$ ) activity each week ${ }^{(25)}$. We also assessed the effects of the diets on mood using the Profile of Mood States questionnaire ${ }^{(39)}$, and asked women to report any adverse or positive physical or psychosocial effects of the diets throughout the 4-month trial.

\section{Statistical analyses}

The sample size of thirty-eight subjects/group was initially chosen to detect a difference of 0.8 unit HOMA insulin resistance between the IECR and DER groups (corresponding to a $50 \%$ decrease in IECR $v$. a $5 \%$ decrease in DER) with $80 \%$ power (assuming a standard deviation of $1 \cdot 1$ unit), allowing for a $20 \%$ dropout as a per-protocol analysis. We considered it more appropriate to report a comparison of the three dietary groups (adjusting for multiple testing with Bonferroni correction) with an intention-to-treat analysis that includes all subjects in a last-observation-carried-forward (LOCF) analysis. This comparison has $80 \%$ power to detect differences of $0 \cdot 8$ or more.

Data at baseline, 1, 3 and 4 months are presented as means (95\% CI) or geometric means (95\% CI) for log-transformed variables (fasting insulin, insulin resistance, HbA1c, adiponectin, leptin, leptin:adiponectin ratio, total IGF-1 and dietary parameters), or medians (95\% CI) for non-parametric variables (plasma IL-6, TNF- $\alpha$, alcohol, physical activity and systolic blood pressure). The primary analysis was an intentionto-treat LOCF ANCOVA at 3 months between the groups defined at randomisation adjusted for the baseline level of each parameter. We also conducted a sensitivity analysis of women who completed the 4-month study. This showed the same magnitude of differences as the LOCF analysis, although, as expected, due to the smaller numbers, differences were no longer statistically significant. Statistical significance was accepted at $P<0.05$. Data were analysed using SPSS (version 15; SPSS Limited). 


\section{Results}

\section{Study population}

Baseline demographic and clinical characteristics of the three groups of randomised women are reported in Table 1 . The groups were of comparable age, menopausal status and similar with respect to other demographic characteristics; 97\% were Caucasian. Of the study participants, nine IECR (24\%), twelve IECR + PF (32\%) and thirteen DER (33\%) participants had insulin resistance at baseline defined as a HOMA score of $>2.5$ unit $(P=0.71)^{(40)}$, while six IECR (16\%), eight IECR + PF (21\%) and nine DER (23\%) met the revised harmonised criteria for the metabolic syndrome $(P=0.65)^{(41)}$. The majority of the subjects reported previous attempts to the diet (IECR $86 \%$, IECR + PF $90 \%$ and DER $95 \%$ of the participants), with a comparable number of previous attempts between the groups (IECR: median 2.0 (inter-quartile range $1 \cdot 0-5 \cdot 0$ ); IECR + PF: median 2.0 (inter-quartile range 1.0-4.4); DER: median $2 \cdot 0$ (inter-quartile range $1 \cdot 0-5 \cdot 0$ ); $P=0.59$ ).

In total, twenty-seven women withdrew from the study (23\%): IECR $n 4$ (11\%), IECR + PF $n 10$ (26\%) and DER $n 13(33 \%)\left(\chi^{2}=5 \cdot 3, P=0 \cdot 071\right)$. The reasons for the dropout were family/work issues (IECR $n 3$, IECR + PF $n 4$ and DER $n$ 5), unrelated personal illness (IECR $n 1$, IECR + PF

Table 1. Baseline characteristics of the subjects

(Mean values and standard deviations; number of subjects and percentages)

\begin{tabular}{|c|c|c|c|c|c|c|}
\hline & \multicolumn{2}{|c|}{$\begin{array}{l}\text { IECR } \\
\left(\begin{array}{l}n 37)\end{array}\right.\end{array}$} & \multicolumn{2}{|c|}{$\begin{array}{c}\mathrm{IECR}+\mathrm{PF} \\
\quad(n 38)\end{array}$} & \multicolumn{2}{|c|}{$\begin{array}{l}\text { DER } \\
(n 40)\end{array}$} \\
\hline & $n$ & $\%$ & $n$ & $\%$ & $n$ & $\%$ \\
\hline \multicolumn{7}{|l|}{ Age at start (years) } \\
\hline Mean & \multicolumn{2}{|c|}{$45 \cdot 6$} & \multicolumn{2}{|c|}{$48 \cdot 6$} & \multicolumn{2}{|c|}{47.9} \\
\hline SD & \multicolumn{2}{|c|}{$8 \cdot 3$} & \multicolumn{2}{|c|}{$7 \cdot 3$} & \multicolumn{2}{|c|}{$7 \cdot 7$} \\
\hline \multicolumn{7}{|l|}{ Baseline BMI $\left(\mathrm{kg} / \mathrm{m}^{2}\right)$} \\
\hline Mean & \multirow{2}{*}{\multicolumn{2}{|c|}{$\begin{array}{c}29.6 \\
4.1\end{array}$}} & \multicolumn{2}{|c|}{$31 \cdot 0$} & \multicolumn{2}{|c|}{$32 \cdot 2$} \\
\hline SD & & & \multicolumn{2}{|c|}{$5 \cdot 7$} & \multicolumn{2}{|c|}{5.6} \\
\hline Breast cancer risk $(\%)^{*}$ & 27 & 9 & 27 & 9 & 27 & 10 \\
\hline \multicolumn{7}{|l|}{ Social circumstances } \\
\hline Married or cohabiting & 26 & $72 \cdot 2$ & 32 & 88.9 & 32 & $82 \cdot 1$ \\
\hline Children living at home & 26 & $70 \cdot 3$ & 24 & $66 \cdot 7$ & 30 & 76.9 \\
\hline \multicolumn{7}{|l|}{ Employment } \\
\hline Full time & 29 & 78.4 & 20 & $52 \cdot 6$ & 25 & 62.5 \\
\hline Part time & 4 & $10 \cdot 8$ & 11 & 28.9 & 7 & $17 \cdot 5$ \\
\hline Retired or unemployed & 4 & $10 \cdot 8$ & 7 & $18 \cdot 4$ & 8 & $20 \cdot c$ \\
\hline Current smoker & 4 & $11 \cdot 1$ & 3 & 7.9 & 4 & $10 \cdot c$ \\
\hline \multicolumn{7}{|l|}{ Medication } \\
\hline Anti-inflammatories & 2 & 5.4 & 0 & 0.0 & 4 & 10 \\
\hline Statins & 1 & $2 \cdot 7$ & 1 & $2 \cdot 6$ & 3 & 7.5 \\
\hline CVD medication & 4 & $10 \cdot 8$ & 5 & $13 \cdot 2$ & 4 & $10 \cdot c$ \\
\hline Antidepressants & 3 & $8 \cdot 1$ & 3 & 7.9 & 7 & 17.5 \\
\hline Thyroxin & 1 & $2 \cdot 7$ & 1 & $2 \cdot 6$ & 2 & $5 \cdot 0$ \\
\hline Contraceptives & 6 & $16 \cdot 2$ & 3 & 7.9 & 2 & 5.0 \\
\hline HRT & 5 & 13.5 & 3 & 7.9 & 5 & 12.5 \\
\hline \multicolumn{7}{|l|}{ Menopausal status } \\
\hline Pre & 18 & $50 \cdot 0$ & 16 & $47 \cdot 1$ & 18 & 47.4 \\
\hline Peri & 3 & 8.3 & 3 & 8.8 & 3 & 7.9 \\
\hline Post & 15 & $41 \cdot 7$ & 15 & 44.1 & 17 & 44.7 \\
\hline
\end{tabular}

IECR, intermittent energy and carbohydrate restriction; IECR + PF, IECR + ad libitum protein and fat; DER, daily energy restriction; HRT, hormone replacement therapy.

* Lifetime risk; Tyrer-Cuzick model ${ }^{(24)}$. $n 1$ and DER $n$ 1), problems adhering to the diet (IECR + PF $n 2$ and DER $n$ 3) and loss to follow up (IECR + PF $n 3$ and DER $n$ 4) (Fig. 1).

\section{Effects of the diets during the 3-month weight-loss period}

Changes in insulin sensitivity and disease risk markers. At 3 months, the IECR group had significantly greater reductions in serum insulin $(P=0.017)$ and HOMA $(P=0.02)$ from baseline values compared with the DER group. Similar reductions were also observed with the IECR + PF group, but were not significantly greater than with the DER group (insulin $P=0.176$ ) and HOMA $P=0 \cdot 210$; Table 2 ). Both IECR groups experienced further acute reductions in insulin resistance during their $2 \mathrm{~d}$ restriction. At 3 months, the median percentage reduction in HOMA from immediately before their $2 \mathrm{~d}$ restriction (after $5 \mathrm{~d}$ of the unrestricted diet) to the morning immediately after the $2 \mathrm{~d}$ restriction was -40 (interquartile range -62 to -45$) \%$ for the IECR group ( $n$ 6) and -27 (interquartile range -14.5 to +12$) \%$ for the IECR $+\mathrm{PF}$ group ( $n$ 5) (Fig. S1, available online). The concentration of HbA1c, a measure of long-term plasma glucose concentrations, was not significantly different between the three dietary groups.

At 3 months, all groups experienced small increases in the levels of the ketone $\beta$-hydroxybutyrate when measured in both plasma (Table 2) and urine samples (data not shown). Plasma $\beta$-hydroxybutyrate was additionally elevated after the $2 \mathrm{~d}$ restriction. At 3 months, the median percentage increase in $\beta$-hydroxybutyrate from before their $2 \mathrm{~d}$ restriction (after $5 \mathrm{~d}$ of the unrestricted diet) to the morning immediately after the $2 \mathrm{~d}$ restriction was 31 (interquartile range -22 to +75$) \%$ for the IECR group $(n 6)$ and 78 (interquartile range +26 to $+98) \%$ for the IECR + PF group ( $n$ 5) (Fig. S1, available online). At 3 months, all dietary groups experienced comparable decreases in serum leptin, the leptin:adiponectin ratio, reductions and the inflammatory marker IL- 6 but no significant changes in serum adiponectin, IGF-1, TNF- $\alpha$ or advanced oxidation protein products (a measure of systemic oxidative stress), when measured in plasma (Table 2) or urine samples (data not shown). All groups experienced small and comparable reductions in total and LDL-cholesterol and TAG, maintenance of HDL-cholesterol, a small decrease in the total:HDL-cholesterol ratio and reduced systolic and diastolic blood pressure (Table 2). Both IECR diets evoked an acute reduction in TAG. At 3 months, the median percentage decrease from immediately before their $2 \mathrm{~d}$ restriction (after $5 \mathrm{~d}$ of the unrestricted diet) to the morning immediately after the $2 \mathrm{~d}$ restriction for the IECR group was -6 (interquartile range -32 to +4$) \%(n 6)$ and for the IECR + PF group -14 (inter-quartile range $-28-11) \%$. The $2 \mathrm{~d}$ restriction did not evoke consistent acute changes in any of the other metabolic endpoints assessed in the study (Fig. S1, available online).

Changes in weight, adiposity and body circumferences. The proportions of the three groups achieving $5 \%$ or greater weight loss at 3 months were $65 \%$ (IECR), 58\% (IECR + PF) and $40 \%$ (DER) $\left(\chi^{2}=5 \cdot 2, P=0 \cdot 076\right)$. Both IECR groups experienced significantly greater and comparable reductions 
NS British Journal of Nutrition

Table 2. Insulin and other disease risk biomarkers over 4 months

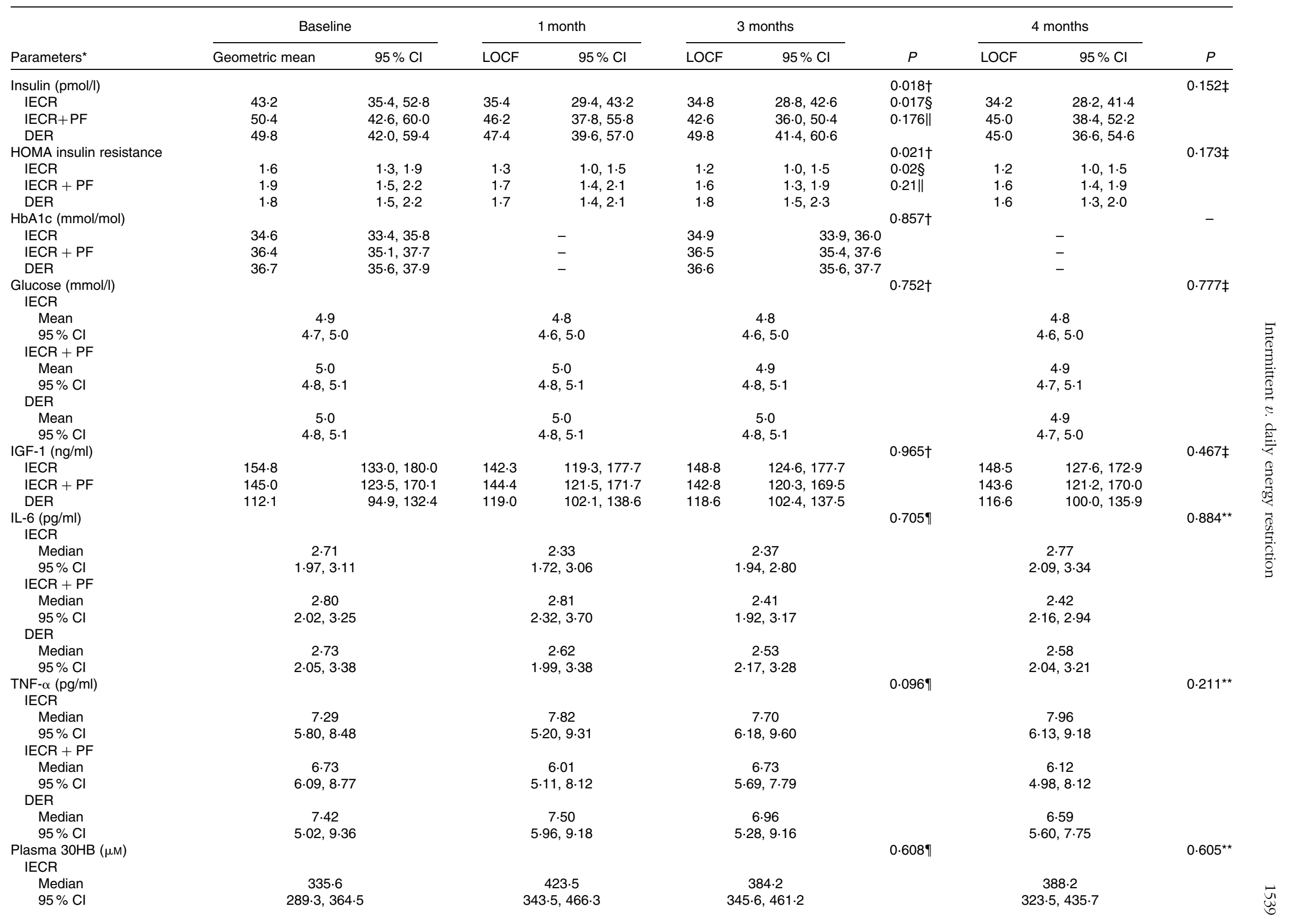


Nesitish Journal of Nutrition

Table 2. Continued

\begin{tabular}{|c|c|c|c|c|c|c|c|c|c|c|}
\hline \multirow[b]{2}{*}{ Parameters* } & \multicolumn{2}{|c|}{ Baseline } & \multicolumn{2}{|c|}{1 month } & \multicolumn{2}{|c|}{3 months } & \multirow[b]{2}{*}{$P$} & \multicolumn{2}{|c|}{4 months } & \multirow[b]{2}{*}{$P$} \\
\hline & Geometric mean & $95 \% \mathrm{Cl}$ & LOCF & $95 \% \mathrm{Cl}$ & LOCF & $95 \% \mathrm{Cl}$ & & LOCF & $95 \% \mathrm{Cl}$ & \\
\hline \multicolumn{11}{|l|}{$\mathrm{IECR}+\mathrm{PF}$} \\
\hline Median & \multirow{2}{*}{\multicolumn{2}{|c|}{$\begin{array}{c}349.7 \\
303.7 .391 .1\end{array}$}} & \multirow{2}{*}{\multicolumn{2}{|c|}{$\begin{array}{c}387.0 \\
352.7 .493 .0\end{array}$}} & \multirow{2}{*}{\multicolumn{2}{|c|}{$\begin{array}{c}357.5 \\
315.4 .422 .9\end{array}$}} & \multirow{2}{*}{\multicolumn{4}{|c|}{$\begin{array}{c}352 \cdot 7 \\
323 \cdot 2,384 \cdot 0\end{array}$}} \\
\hline $95 \% \mathrm{Cl}$ & & & & & & & & & & \\
\hline \multicolumn{11}{|l|}{ DER } \\
\hline Median & \multirow{2}{*}{\multicolumn{2}{|c|}{$\begin{array}{c}341 \cdot 7 \\
315 \cdot 9,357 \cdot 6\end{array}$}} & \multirow{2}{*}{\multicolumn{2}{|c|}{$\begin{array}{c}369.2 \\
340 \cdot 5,399.0\end{array}$}} & \multirow{2}{*}{\multicolumn{2}{|c|}{$\begin{array}{c}339.7 \\
318 \cdot 4,376 \cdot 7\end{array}$}} & \multicolumn{4}{|c|}{359.0} \\
\hline $95 \% \mathrm{Cl}$ & & & & & & & \multirow{3}{*}{\multicolumn{3}{|c|}{$332 \cdot 9,395 \cdot 4$}} & \\
\hline Plasma AOPP $(\mu \mathrm{M})$ & & & & & & & & & & $0.087 \ddagger$ \\
\hline IECR & & & & & & & & & & \\
\hline Mean & & & & & & & & & & \\
\hline $95 \% \mathrm{Cl}$ & 221.5 & & & 344.2 & & 332.6 & & & 348.4 & \\
\hline $\mathrm{IECR}+\mathrm{PF}$ & & & & & & & & & & \\
\hline Mean & & & & & & & & & & \\
\hline $95 \% \mathrm{Cl}$ & $262 \cdot \varepsilon$ & & & $368 \cdot 7$ & & 384.0 & & & 369.9 & \\
\hline DER & & & & & & & & & & \\
\hline Mean & & & & & & & & & & \\
\hline $95 \% \mathrm{Cl}$ & 265.4 & & & $344 \cdot 6$ & & $369 \cdot 1$ & & & 329.4 & \\
\hline Leptin (ng/ml) & & & & & & & $0.075 t$ & & & $0.057 \ddagger$ \\
\hline IECR & $29 \cdot 3$ & $24 \cdot 6,34.9$ & 18.4 & $14 \cdot 9,22 \cdot 8$ & $16 \cdot 7$ & $13.4,20.9$ & & $16 \cdot 3$ & $12 \cdot 8,20 \cdot 7$ & \\
\hline $\mathrm{IECR}+\mathrm{PF}$ & 34.8 & $28 \cdot 0,43 \cdot 3$ & 24.0 & $18 \cdot 1,31.9$ & $22 \cdot 4$ & $16 \cdot 9,29 \cdot 5$ & & $24 \cdot 8$ & $19 \cdot 3,31 \cdot 8$ & \\
\hline DER & 35.5 & $29 \cdot 9,42 \cdot 2$ & $25 \cdot 0$ & $20 \cdot 1,31 \cdot 0$ & $25 \cdot 4$ & $19 \cdot 9,32 \cdot 4$ & & $26 \cdot 5$ & $20 \cdot 9,33.6$ & \\
\hline Adiponectin $(\mu \mathrm{g} / \mathrm{ml})$ & & & & & & & $0.464 \dagger$ & & & $0.456 \ddagger$ \\
\hline IECR & $13 \cdot 8$ & $9 \cdot 8,19 \cdot 3$ & $12 \cdot 2$ & $8 \cdot 4,17 \cdot 6$ & 13.6 & $9 \cdot 7,19 \cdot 0$ & & $15 \cdot 0$ & $10 \cdot 8,20 \cdot 8$ & \\
\hline $\mathrm{IECR}+\mathrm{PF}$ & $13 \cdot 1$ & $9 \cdot 0,19 \cdot 2$ & 12.4 & $8.5,17.9$ & $13 \cdot 2$ & $9 \cdot 4,18 \cdot 6$ & & $12 \cdot 7$ & $9 \cdot 0,17 \cdot 8$ & \\
\hline DER & 11.2 & $8.5,14.8$ & 9.0 & $6 \cdot 5,12 \cdot 4$ & 10.2 & $7 \cdot 8,13 \cdot 2$ & & 10.6 & $8 \cdot 1,13.7$ & \\
\hline Leptin:adiponectin ratio & & & & & & & $0.325 \rrbracket$ & & & $0.094^{* *}$ \\
\hline IECR & & & & & & & & & & \\
\hline Median & & & & & & & & & & \\
\hline $95 \% \mathrm{Cl}$ & & & & & & 1.3 & & & & \\
\hline $\mathrm{IECR}+\mathrm{PF}$ & & & & & & & & & & \\
\hline Median & & & & & & & & & & \\
\hline $95 \% \mathrm{Cl}$ & & & & & & 1.6 & & & & \\
\hline DER & & & & & & & & & & \\
\hline Median & & & & & & & & & & \\
\hline $95 \% \mathrm{Cl}$ & & & & & & 1.7 & & & & \\
\hline Cholesterol ( $\mathrm{mmol} / \mathrm{l})$ & & & & & & & $0.587 \dagger$ & & & $0.835 \ddagger$ \\
\hline IECR & & & & & & & & & & \\
\hline Mean & & & & & & & & & & \\
\hline $95 \% \mathrm{Cl}$ & 4.95 & & & $5 \cdot 14$ & & $5 \cdot 35$ & & & 5.42 & \\
\hline $\mathrm{IECR}+\mathrm{PF}$ & & & & & & & & & & \\
\hline Mean & & & & & & & & & & \\
\hline $95 \% \mathrm{Cl}$ & 5.33 & & & 5.74 & & 5.90 & & & $5 \cdot 88$ & \\
\hline DER & & & & & & & & & & \\
\hline Mean & & & & & & & & & & \\
\hline $95 \% \mathrm{Cl}$ & 4.95 & & & $5 \cdot 37$ & & 5.46 & & & 5.52 & \\
\hline $\mathrm{TAG}(\mathrm{mmol} / \mathrm{l})$ & & & & & & & $0.243 \dagger$ & & & $0.356 \ddagger$ \\
\hline IECR & 0.99 & $0.85,1.15$ & 0.84 & $0.74,0.94$ & 0.85 & $0.74,0.97$ & & 0.90 & $0.80,1.03$ & \\
\hline $\mathrm{IECR}+\mathrm{PF}$ & 1.07 & $0.92,1.23$ & 0.95 & $0.83,1.08$ & 0.94 & $0.81,1.08$ & & 0.92 & $0.80,1.05$ & \\
\hline DER & 1.09 & $0.94,1.25$ & 1.00 & $0.87,1.15$ & 1.01 & $0.89,1.16$ & & 1.01 & $0.89,1.16$ & \\
\hline
\end{tabular}


Table 2. Continued

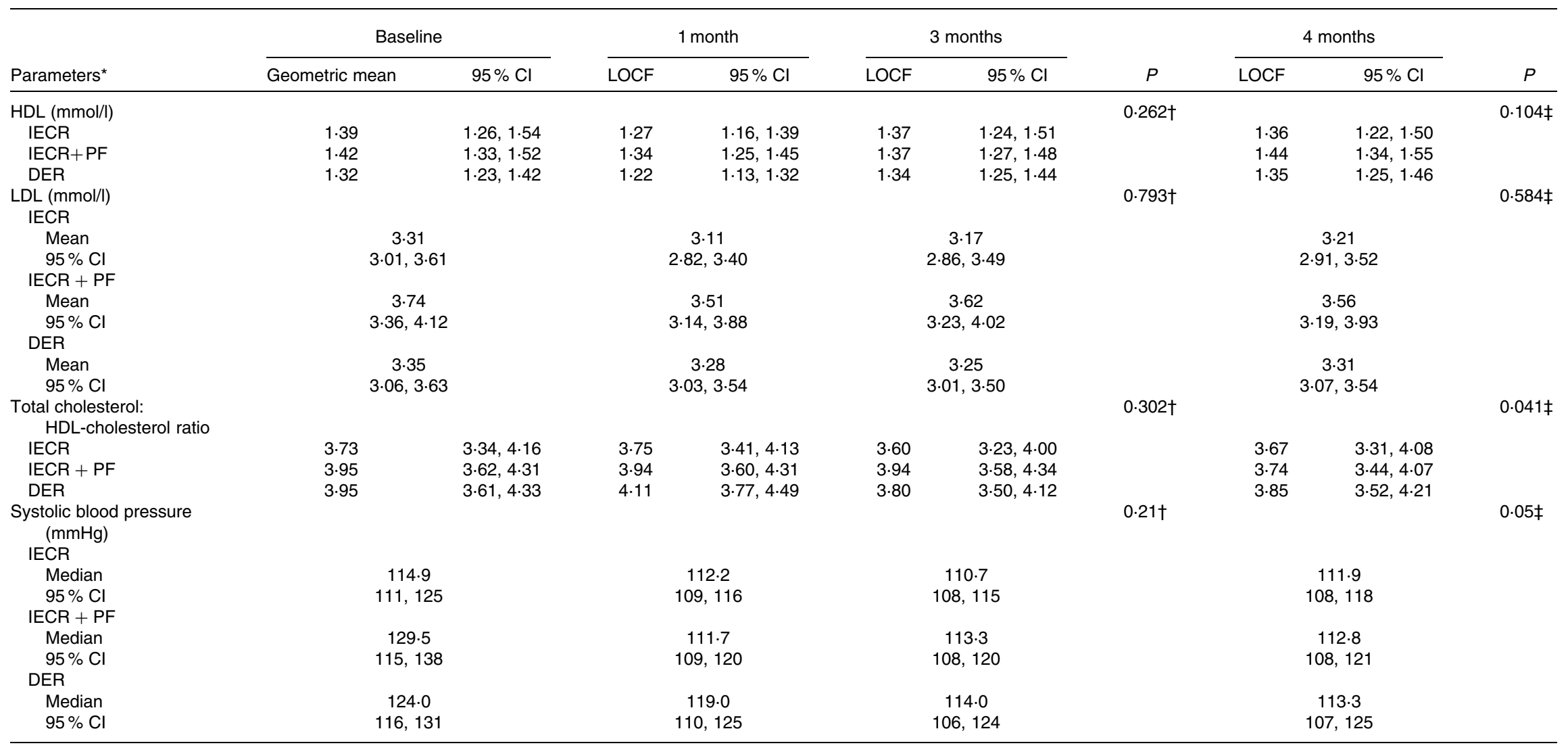

LOCF, last observation carried forward; IECR, intermittent energy and carbohydrate restriction; IECR + PF, IECR + ad libitum protein and fat; DER, daily energy restriction; HOMA, homeostasis model assessment; HbA1c, glycated $\mathrm{Hb}$; IGF-1, insulin-like growth factor 1 ; $30 \mathrm{HB}, \beta$-hydroxybutyrate; AOPP, advanced oxidation protein products.

*IECR ( $n$ 37), IECR + PF $(n 38)$ and DER ( $n 40)$.

TANCOVA between the three groups for LOCF at 3 months adjusted for baseline values.

ҒANCOVA between the three groups for LOCF at 4 months adjusted for 3-month LOCF values.

$\S$ ANCOVA between IECR and DER for LOCF at 3 months adjusted for baseline values with Bonferroni adjustment.

$\|$ ANCOVA between IECR + PF and DER for LOCF at 3 months adjusted for baseline values.

9 Kruskal-Wallis between the three groups for the change from baseline to LOCF at 3 months.

** Kruskal-Wallis between the three groups for the change from LOCF at 3 months to LOCF at 4 months. 
in body fat than the DER group (IECR $P=0.007$ and IECR + PF $P=0.019$ ), but no significantly greater reductions in weight, waist, hip and bust circumferences (Table 3). There were small reductions in FFM in all the three groups. The median percentage of weight lost, which was FFM, was less in the IECR + PF group $(20 \cdot 4$ (95\% CI 13.2, 27.2) \%) compared with both IECR $(36.0$ (95\% CI 26.4, 41.3)\%,P=0.009) and DER (29.3 (95\% CI 25, 38.1) \%, $P=0 \cdot 048)$ groups.

\section{Dietary adherence}

The mean percentage of the potential two restricted days per week completed during the 3-month weight-loss period was reported to be $76(95 \%$ CI 67, 85) \% for the IECR group and $74(95 \%$ CI 64, 84) \% for the IECR + PF group, with the majority as two consecutive days (IECR $86 \%$ and IECR + PF 94\%) and the remainder as individual days.

Weekly average energy, carbohydrate, total fat, SFA and alcohol intakes were reduced in all the diet groups (LOCF analysis). The reductions in carbohydrate were greatest in the IECR and IECR + PF groups: on average, the IECR and IECR + PF groups both reduced carbohydrate by $43 \%$ compared with $23 \%$ for the DER group. The IECR + PF group maintained higher intakes of protein and fat compared with the other groups with, on average, an additional $10 \mathrm{~g}$ of protein and $15 \mathrm{~g}$ of fat per $\mathrm{d}$ when estimated after 3 months (Table S1, available online).

Both IECR groups were instructed to have a euenergetic diet during the $5 \mathrm{~d}$ periods between the restrictions but, instead, had a 'carry-over effect' of the reduced intake of energy and carbohydrate on these non-restricted days, which was significantly lower than the diet that we prescribed. At 3 months, the mean energy intake for a 'non-restricted' day of the IECR group was 5716 (95\% CI 5215, 6216) kJ compared with their prescribed diet of $8314(95 \%$ CI 8080, 8540) kJ, on average $32 \%$ less than prescribed. Likewise, the mean energy intake for a non-restricted day of the IECR + PF group was 6439 (95\% CI 5878, 7000) kJ compared with their prescribed diet of $8458(95 \% \mathrm{CI} 8193,8722) \mathrm{kJ}$, on average $23 \%$ less than prescribed. The mean carbohydrate intakes on non-restricted days of the IECR and IECR + PF groups were $152 \cdot 3$ (95\% CI $135 \cdot 1,169 \cdot 4)$ and 154.9 (95\% CI 139.1, 170.7) g, respectively, compared with the prescribed 223.5 (95\% CI 217.2, 229.8) and $227 \cdot 4$ (95\% CI $220 \cdot 2,234 \cdot 5) \mathrm{g}$, both $32 \%$ less than prescribed (all $P<0.001$ ). The mean energy and carbohydrate intakes on non-restricted days of the IECR and IECR + PF groups were actually comparable with the daily restricted group, who had the mean daily intakes of 6126.7 (95\% CI $5575,6678) \mathrm{kJ}$ and $169 \cdot 5$ (95\% CI 155.1, 183.4) g carbohydrate (both $P=1 \cdot 00$ ). Physical activity diaries documented minimal

Table 3. Weight, adiposity and circumferences over 4 months

(Mean values and $95 \%$ confidence intervals)

\begin{tabular}{|c|c|c|c|c|c|c|c|c|c|c|}
\hline \multirow[b]{2}{*}{ Parameters ${ }^{\star}$} & \multicolumn{2}{|c|}{ Baseline } & \multicolumn{2}{|c|}{1 month } & \multicolumn{2}{|c|}{3 months } & \multirow[b]{2}{*}{$P$} & \multicolumn{2}{|c|}{4 months } & \multirow[b]{2}{*}{$P$} \\
\hline & Mean & $95 \% \mathrm{Cl}$ & Mean & $95 \% \mathrm{Cl}$ & Mean & $95 \% \mathrm{Cl}$ & & Mean & $95 \% \mathrm{Cl}$ & \\
\hline Weight $(\mathrm{kg}) \dagger$ & & & & & & & $0.10 \ddagger$ & & & $0.54 \S$ \\
\hline IECR & $79 \cdot 4$ & $74 \cdot 6,84 \cdot 1$ & $77 \cdot 0$ & $72 \cdot 4,81.5$ & 74.4 & $70 \cdot 0,78.9$ & & 73.9 & $69 \cdot 4,78.5$ & \\
\hline IECR+PF & 82.4 & $77 \cdot 2,87 \cdot 6$ & $80 \cdot 1$ & $75 \cdot 2,84.9$ & $77 \cdot 6$ & $72 \cdot 9,82 \cdot 4$ & & $77 \cdot 3$ & $72 \cdot 5,82 \cdot 1$ & \\
\hline DER & $86 \cdot 0$ & $80 \cdot 6,91 \cdot 3$ & 83.7 & $78 \cdot 5,88 \cdot 8$ & $82 \cdot 3$ & $77 \cdot 1,87 \cdot 5$ & & $82 \cdot 2$ & $76 \cdot 9,87.5$ & \\
\hline Body fat $(\mathrm{kg}) \dagger$ & & & & & & & $0.004 \ddagger$ & & & $0.659 \S$ \\
\hline IECR & 31.0 & $27 \cdot 9,34 \cdot 2$ & $29 \cdot 3$ & $26 \cdot 5,32 \cdot 1$ & $27 \cdot 3$ & $24 \cdot 6,30 \cdot 0$ & $0.007 \|$ & $26 \cdot 7$ & $23 \cdot 9,29 \cdot 5$ & \\
\hline $\mathrm{IECR}+\mathrm{PF}$ & 33.5 & $29 \cdot 9,37 \cdot 0$ & $31 \cdot 6$ & $28 \cdot 3,35 \cdot 0$ & $29 \cdot 7$ & $26 \cdot 6,32 \cdot 9$ & 0.019 q & 29.4 & $26 \cdot 3,32 \cdot 6$ & \\
\hline DER & 35.7 & $32 \cdot 3,39 \cdot 2$ & 34.6 & $31 \cdot 2,38 \cdot 0$ & 33.7 & $30 \cdot 3,37 \cdot 2$ & & $33 \cdot 2$ & $29 \cdot 7,36 \cdot 7$ & \\
\hline Fat-free mass $(\mathrm{kg}) \dagger$ & & & & & & & $0.288 \ddagger$ & & & $0.297 \S$ \\
\hline IECR & 48.5 & $46 \cdot 4,50.5$ & 47.6 & $45 \cdot 6,49 \cdot 7$ & $46 \cdot 7$ & $44 \cdot 4,49 \cdot 0$ & & $47 \cdot 2$ & $45 \cdot 1,49 \cdot 3$ & \\
\hline IECR + PF & $49 \cdot 0$ & $47 \cdot 2,50 \cdot 9$ & 48.5 & $46 \cdot 7,50 \cdot 3$ & 47.9 & $46 \cdot 2,49 \cdot 7$ & & $47 \cdot 9$ & $46 \cdot 1,49 \cdot 6$ & \\
\hline DER & $50 \cdot 3$ & $48 \cdot 2,52 \cdot 3$ & $49 \cdot 2$ & $47 \cdot 4,51 \cdot 1$ & 48.9 & $47 \cdot 1,50 \cdot 8$ & & $48 \cdot 7$ & $46 \cdot 5,50 \cdot 8$ & \\
\hline Waist $(\mathrm{cm}) \dagger$ & & & & & & & $0.088 \ddagger$ & & & $0.475 \S$ \\
\hline IECR & $100 \cdot 5$ & $96 \cdot 6,104 \cdot 5$ & $98 \cdot 6$ & $94 \cdot 7,102 \cdot 5$ & $95 \cdot 2$ & $91 \cdot 4,99 \cdot 1$ & & 94.4 & $90 \cdot 5,98 \cdot 3$ & \\
\hline $\mathrm{IECR}+\mathrm{PF}$ & $104 \cdot 1$ & $99 \cdot 0,109 \cdot 1$ & $101 \cdot 8$ & $96 \cdot 9,106 \cdot 7$ & $99 \cdot 3$ & $94 \cdot 5,104 \cdot 2$ & & $98 \cdot 8$ & $94 \cdot 1,103 \cdot 6$ & \\
\hline DER & $106 \cdot 0$ & $101 \cdot 9,110 \cdot 2$ & $103 \cdot 8$ & $99 \cdot 8,107 \cdot 8$ & $102 \cdot 7$ & $98 \cdot 4,107 \cdot 0$ & & $102 \cdot 4$ & $98 \cdot 0,106 \cdot 8$ & \\
\hline Hip (cm)† & & & & & & & $0.144 \ddagger$ & & & $0.169 \S$ \\
\hline IECR & $108 \cdot 8$ & $105 \cdot 9,111 \cdot 7$ & $107 \cdot 4$ & $104 \cdot 4,110 \cdot 4$ & $104 \cdot 8$ & $101 \cdot 6,108 \cdot 0$ & & $103 \cdot 7$ & $100 \cdot 2,107 \cdot 3$ & \\
\hline $\mathrm{IECR}+\mathrm{PF}$ & 111.0 & $107 \cdot 3,114 \cdot 6$ & $108 \cdot 9$ & $105 \cdot 7,112 \cdot 1$ & $107 \cdot 1$ & $103 \cdot 7,110 \cdot 4$ & & $106 \cdot 7$ & $103 \cdot 2,110 \cdot 2$ & \\
\hline DER & $112 \cdot 6$ & $109 \cdot 2,116.0$ & $111 \cdot 1$ & $107 \cdot 8,114.5$ & 109.8 & $106 \cdot 2,113 \cdot 3$ & & $109 \cdot 8$ & $106 \cdot 1,113 \cdot 4$ & \\
\hline Bust $(\mathrm{cm}) \dagger$ & & & & & & & $0.098 \ddagger$ & & & $0.418 \S$ \\
\hline IECR & $102 \cdot 8$ & $99.6,105.9$ & $101 \cdot 3$ & $98 \cdot 1,104 \cdot 4$ & 99.4 & $96 \cdot 3,102 \cdot 5$ & & $98 \cdot 6$ & $95.4,101.8$ & \\
\hline IECR + PF & $104 \cdot 6$ & $100 \cdot 9,108 \cdot 4$ & $102 \cdot 7$ & $99 \cdot 1,106 \cdot 3$ & 101.4 & $97 \cdot 6,105 \cdot 1$ & & $101 \cdot 1$ & $97 \cdot 4,104 \cdot 8$ & \\
\hline DER & 108.5 & $105 \cdot 2,111 \cdot 7$ & $106 \cdot 7$ & $103 \cdot 4,110 \cdot 1$ & $106 \cdot 2$ & $102 \cdot 9,109.5$ & & $105 \cdot 9$ & $102 \cdot 5,109.3$ & \\
\hline
\end{tabular}

IECR, intermittent energy and carbohydrate restriction; IECR + PF, IECR + ad libitum protein and fat; DER, daily energy restriction.

* IECR ( $n$ 37), IECR + PF ( $n$ 38) and DER ( $n$ 40).

† Baseline and last-observation-carried-forward (LOCF) values at 1, 3 and 4 months.

$\ddagger$ ANCOVA for LOCF between the three groups at 3 months adjusted for baseline values.

$\S$ ANCOVA for LOCF between the three groups at 4 months adjusted for 3-month LOCF values.

$\|$ ANCOVA for LOCF at 3 months between IECR and DER adjusted for baseline values with Bonferroni adjustment.

I ANCOVA for LOCF at 3 months between IECR + PF and DER adjusted for baseline values with Bonferroni adjustment. 
changes in activity with no significant differences between the three dietary groups (Table S1, available online).

\section{Acceptability and safety of the diets}

No major adverse effects were reported. A small number of women in the three diet groups reported minor physical symptoms such as feeling cold (DER 3\%), decreased energy levels (IECR 5\% and DER 5\%), constipation (IECR 8\% and DER 3\%), headaches (IECR 5\% and IECR + PF 3\%), bad breath on energy-restricted days (IECR 5\% and IECR + PF $3 \%$ ), feeling light-headed (IECR 3\%), lack of concentration (DER 3\%), mood swings or bad temper (IECR 3\% and DER 5\%) and being preoccupied with food (IECR $8 \%$, IECR + PF 3\% and DER 3\%).

Average weekly hunger scores did not differ between the three groups throughout the study. At 1 month, hunger and desire-to-eat scores were marginally greater after the first restricted day of IECR compared with the first restricted day of IECR $+\mathrm{PF}$ and an average DER day (both $P<0.05$ ). This difference was no longer evident after 3 and 4 months, indicating that women had become used to the restriction (Table S2, available online). All groups experienced comparable reductions in Profile of Mood States questionnaire scores for tension, depression, anger, fatigue and confusion, an increase in vigour and an overall decline in total mood disturbance (Table S3, available online).

All diets met requirements for $\mathrm{B}$ vitamins, vitamin $\mathrm{E}$ and vitamin $\mathrm{C}$, but indicated deficiencies in $\mathrm{Mg}, \mathrm{Fe}, \mathrm{Zn}, \mathrm{Ca}$, vitamins $\mathrm{A}$ and $\mathrm{D}$ and fibre as commonly seen with energyreduced diets ${ }^{(42)}$. The deficiency of $\mathrm{Mg}, \mathrm{Zn}$ and Se was most apparent in the IECR group, while inadequate fibre intake was most apparent in the IECR and IECR + PF groups (Table S4(a) and (b), available online).

\section{Weight maintenance period between 3 and 4 months}

Mean adherence to the $1 \mathrm{~d}$ restriction during the weight maintenance month was 68 (95\% CI 50-85) \% for the IECR group and $68(95 \%$ CI 51-87) \% for the IECR + PF group. The reductions in insulin resistance, weight, adiposity, circumferences, leptin, IL-6 and lipids were maintained during the final weight maintenance month in all groups (Tables 2 and 3). Thus, $1 \mathrm{~d}$ of restriction per week maintained weight loss and the reductions in disease risk markers in the short term, similarly to the DER group who were asked to have the recalculated euenergetic DER.

\section{Discussion}

The present randomised trial indicates that, in the short term, the IECR diet is superior to the DER diet with respect to the improvements in insulin sensitivity and the loss of body fat. There were additional increases in insulin sensitivity with IECR when measured at the end of the $2 \mathrm{~d}$ restriction, although the significance of this additional effect is not clear. Higher serum $\beta$-hydroxybutyrate indicates greater fat oxidation during the $2 \mathrm{~d}$ restriction. IECR with ad libitum protein and fat was tested in an attempt to increase acceptability. The IECR + PF diet achieved greater reductions in body fat and a trend to improved insulin sensitivity both before and after the $2 \mathrm{~d}$ restriction compared with the DER diet, but was not more acceptable than the IECR diet.

The greater reduction in insulin resistance with the IECR $v$. DER diet ( $5 \mathrm{~d}$ after restriction) in the present trial is consistent with greater reductions in adiposity in the IECR group. Despite the apparent comparable loss of body fat, the IECR + PF group had lesser reductions in insulin resistance. The reasons for this are not known. The IECR + PF diet was higher in protein, total fat and MUFA than the IECR diet (81 $v .71 \mathrm{~g}$ protein per d). Much higher protein intakes $(>140 \mathrm{~g} / \mathrm{d})$ have been linked to increased insulin resistance, particularly alongside low fibre intakes ( $15 \mathrm{~g}$ or less), possibly since amino acids activate the protein ribosomal subunit serine kinase 6-1 that phosphorylates and inhibits the downstream signalling of the insulin receptor. The higher total fat (37\% energy) and MUFA intake (14\% energy) with the IECR + PF diet would be expected to exert either a neutral or beneficial effect on insulin sensitivity ${ }^{(43,44)}$.

Both IECR diets included periods (approximately $60 \mathrm{~h} /$ week) of profound energy and carbohydrate restriction (IECR: $-65 \%$ energy and $-85 \%$ carbohydrate; IECR + PF: $-50 \%$ energy and $-85 \%$ carbohydrate) compared with the DER diet ( $-25 \%$ energy and $-30 \%$ carbohydrate). These spells of energy and carbohydrate restriction evoked acute reductions in insulin resistance and TAG consistent with our previous data for $\mathrm{IER}^{(22)}$, which may be linked to acute reductions in intrahepatic TAG content, improvements in hepatic insulin sensitivity and reduced glucose production ${ }^{(45)}$. The clinical significance of repeated short spells of improved insulin sensitivity is not known. None of the diets altered HbA1c, a longer-term measure of glycaemia. Reduced HbA1c with weight loss has not been consistently demonstrated in non-diabetic populations ${ }^{(46)}$, and may not be sensitive enough to highlight changes in the normoglycaemic population studied here.

Ketones increased during the restricted periods, indicating a switch from glycogenolysis to fat oxidation which may be beneficial, with a potential up-regulation of nuclear and mitochondrial sirtuins, reduced production of reactive oxidative species and reduced accumulation of lipids in skeletal muscle which may protect against metabolic and age-related diseases $^{(47,48)}$

Since IECR and DER have the same overall prescribed energy restriction (25\%), and IECR + PF seemingly a lesser prescribed restriction (15\%), the question arises concerning the mechanism of greater reductions in body fat with the IECR and IECR + PF diets compared with the DER diet. This may be partly due to an apparent greater adherence to the $2 \mathrm{~d}$ of strict restriction with the IECR and IECR + PF diets than to the daily modest energy restriction in the DER group. The present dietary record data show that the percentage of potential restricted days completed during the first month was comparable between the three groups. On average, the IECR and IECR + PF groups completed $80 \%$ of their $2 \mathrm{~d}$ per week during the first month of the diet, while the DER group achieved their $25 \%$ energy restriction goal on 
$80 \%$ of days within their 1 month food diary. By 3 months, the IECR and IECR + PF groups were still undertaking $70 \%$ of their potential restricted days, whereas the DER group achieved their $25 \%$ energy restriction goal on only $39 \%$ of days in their 3-month food diary. While not directly comparable, these estimated values highlight the well-documented poor adherence to DER. Others have reported adherence to DER on $40 \%$ of potential days ${ }^{(49)}$.

The success of both IECR diets is not only linked to the adherence to the $2 \mathrm{~d}$ restriction, but is also attributable to the spontaneous restriction of energy and carbohydrate on non-restricted days. Intakes on these days in the IECR groups were comparable with those of the DER group. This is an important observation and confirms the present data and the data of others which show that intermittent dieting does not lead to disordered eating and overconsumption on non-restricted days ${ }^{(21-23,50,51)}$. This observation would need to be replicated in future studies, but raises the intriguing possibility that IER may be an effective way of achieving short spells of greater restriction as well as a lesser restriction for the remainder of the week.

The apparent comparable loss of body fat between the IECR and IECR + PF groups appears at odds with the higher reported higher energy intake in the IECR + PF group. Part of this may be the slightly higher protein intake with IECR + PF (mean $1.2(95 \%$ CI $1 \cdot 1,1 \cdot 2) \mathrm{g} / \mathrm{kg}$ body weight $v$. mean $1 \cdot 0(95 \%$ CI $0 \cdot 9,1 \cdot 1) \mathrm{g} / \mathrm{kg}$ for the IECR group; mean $1 \cdot 0$ ( $95 \%$ CI $0 \cdot 9,1 \cdot 1) \mathrm{g} / \mathrm{kg}$ for the DER group; $P=0 \cdot 08)$. Although not significantly greater, higher protein intakes will help maximise the loss of fat and limit the loss of FFM for a given energy restriction ${ }^{(52)}$, and may evoke a small increase in energy expenditure and greater fat oxidation ${ }^{(53)}$. Alternatively, the reported higher energy intake with IECR + PF may not actually be higher, and could reflect biased under-reporting of carbohydrate rather than protein foods ${ }^{(54)}$, and thus inflate reported intakes with the higher-protein IECR + PF diet.

The reported $65 \%$ success in producing 5\% weight loss with IECR and $58 \%$ with IECR + PF is encouraging when compared with the $40 \%$ success with DER in the present study and other studies ${ }^{(55)}$. However, this is a short-term study and possibly reflects greater adherence to the novel dietary approach ${ }^{(56)}$. Moreover, three previous short-term randomised trials have tested IER $v$. DER, but unlike the present study they showed no significant differences in weight reduction between the two approaches ${ }^{(21-23)}$. The IER regimens tested here were carbohydrate restricted and higher in protein and fat on restricted days to reduce feelings of hunger ${ }^{(57)}$, which we believe is key for adherence. Our simple assessment of hunger suggests the $2 \mathrm{~d}$ IECR $(65 \%$ energy restriction on restricted days) evoked a modest transient increase in hunger, but hunger did not increase with IECR + PF (50\% energy restriction on restricted days).

The IECR groups consumed carbohydrate on non-restricted days, but did not overconsume, hence our IECR diets were marginally lower in carbohydrate overall than the DER diet. These differences were, however, quite modest with the IECR and IECR + PF groups receiving, respectively, 41 and $37 \%$ of energy overall from carbohydrate compared with $47 \%$ in the DER group. These differences are unlikely to account for the differences in the reductions of weight and adiposity seen ${ }^{(9)}$.

The tested IECR diets meet a number of micronutrient requirements, i.e. thiamin, folic acid and vitamin $\mathrm{C}$, known to be deficient in daily low-carbohydrate diets ${ }^{(42)}$. Suboptimal fibre intake was a particular issue with both IECR diets. The long-term implications of such a low fibre intake are not known; however, comparable intakes found within daily moderate carbohydrate-restricted diets (120-150g/d) have brought about long-term reductions in lipids, homocysteine $^{(58)}$ and the reversal of carotid atherosclerosis over 2 years without detectable adverse effects ${ }^{(27)}$. Future IECR diets should, however, be modified to maximise fibre intake and promote foods rich in $\mathrm{Ca}, \mathrm{Mg}, \mathrm{Fe}$ and $\mathrm{Se}$, which are considered preferable and more physiological than supplements ${ }^{(59)}$, and micronutrient status should be monitored.

Women on both IECR diets were counselled to limit SFA intake, but to include MUFA and PUFA. We reported modest reductions in total and LDL-cholesterol, rather than increased LDL found with long-term high-SFA, low-carbohydrate diets $^{(60)}$. We encouraged a range of lean meat, fish, vegetarian and dairy protein foods in order to discourage high intakes of red meat ( $>500 \mathrm{~g} /$ week), which have been, albeit inconsistently, linked to bowel cancer ${ }^{(61)}$. The IECR + PF diet self-limited to $4776 \mathrm{~kJ}$ and was within healthy limits for protein (i.e. $<125 \mathrm{~g} / \mathrm{d})^{(62)}$ on restricted days and typically included less than $300 \mathrm{~g}$ of red meat/week.

It is well known that maintenance of weight loss presents an even greater challenge than initial weight loss. Including a 1-month weight maintenance period in the present study gives some indication of the relative weight stabilisation effects of the diets. The trial indicates that a $1 \mathrm{~d}$ IECR per week was as effective as DER for maintaining reduced weight and the potential metabolic benefits of weight loss. However, this maintenance period is short and was within a supervised research setting. Further investigation of longer time periods is required.

\section{Limitations}

The $23 \%$ dropout reported here is comparable with many weight loss studies. We present an intention-to-treat analysis based on LOCF. We considered an LOCF analysis the most appropriate method and preferable to other multiple imputation techniques. Dropout from weight loss studies is well known to be linked to poorer success. An LOCF analysis allows all subjects to contribute data and accounts for this potential bias. This would not be achieved using multiple imputation techniques where dropouts are assumed to occur randomly (i.e. observations are assumed to be missing at random). Although randomisation was stratified according to BMI, the IECR group had a lower baseline BMI than the IECR $+\mathrm{PF}(P=0.05)$ and DER groups $(P=0.05)$, which could potentially skew the present findings and partly explain their better outcome. This, however, does not appear to be the case, since baseline BMI did not predict success or dropout; mean LOCF weight loss for women with a BMI above or 
below $30 \mathrm{~kg} / \mathrm{m}^{2}$ was $5.4(\mathrm{SD} 4.2) v .5 .3$ (SD 4.6) \% ( $\left.P=0.874\right)$. The majority of outcomes were assessed by blinded personnel, although weight and anthropometric measures were undertaken by research dietitians not blinded to the diet group. Pre-menopausal women ( $48 \%$ of the cohort) may experience small cyclic variations in the levels of insulin, lipids, leptin and IGF-1 ${ }^{(63,64)}$. We did not time biomarker assessments in relation to the menstrual cycle and were unable to adjust for the day of the cycle in the main analysis.

Body fat and FFM were assessed using bioelectrical impedance. This method is prone to error where there are fluctuations in body water content. However, under the standardised conditions that we used, impedance has been shown to be a valid method for assessing change within weight loss studies with good agreement with appropriate reference methods of assessing body composition such as dual-energy X-ray absorptiometry ${ }^{(65)}$ and multi-compartment models ${ }^{(66)}$.

\section{Implications and future studies}

Intermittent low energy and carbohydrate diets appear feasible in our population of pre- and postmenopausal women. The positive results of the present study warrant further studies to test the veracity of the present short-term results and the longer-term efficacy and safety of the IECR approach and its applicability to other populations. No single dietary approach will be appropriate and feasible for all, and there is unlikely to be a panacea given the complexity of weight management, but the addition of a further choice of intervention may be helpful. In the present trial, eighteen women (12\%) interested in taking part in the study could not adhere to the IECR regimen in a pre-study test of compliance and did not join the study, so the approach will be problematic for some. The potential uptake to the IECR regimen that allows ad libitum protein and fat may be greater but was not assessed. IECR + PF generally produced similar results to IECR except there was a smaller reduction in insulin resistance, but there was a marginally better nutritional profile and a lower proportion of weight loss appeared to be FFM. Further studies of both IECR and IECR + PF regimens are warranted to examine whether they could be a useful alternative dietary intervention for weight-loss programmes.

\section{Supplementary material}

To view supplementary material for this article, please visit http://dx.doi.org/10.1017/S0007114513000792

\section{Acknowledgements}

The present study was supported by the Genesis Breast Cancer Prevention (Registered Charity no. 1109839) and, in part, by the Intramural Research Program of the National Institute on Aging, Baltimore, USA.

The authors would like to thank Helen Sumner for coordinating sample storage and processing, Julie Morris for her invaluable statistical advice, Rosemary Greenhalgh and Jenny Affen for assisting in the recruitment, Jane Eaton, Sue
Rowe and Jayne Beesley for clerical support, Amy Tao, Matthew Collier and Kath Sellers for assisting with the data entry and analysis, and Susan Jebb for her advice on the study design.

The authors' contributions are as follows: M. H. was involved in the trial conception and design, trial management and manuscript preparation; C. W. undertook the role of trial dietitian and participated in the manuscript preparation; $\mathrm{M}$. P. acted as trial dietitian and was involved in the manuscript preparation; D. M. performed the statistical analysis and participated in the manuscript preparation; E. M. conducted the analysis of the diet diaries and assisted in the manuscript preparation; M. P. M. provided consultation, performed assays at the NIH and involved in the manuscript preparation; G. E. was involved in the recruitment of the subjects, consultation and manuscript preparation; B. M. provided consultation, performed leptin, adiponectin, IGF-1, IL-6 and TNF- $\alpha$ assay validation and provided reagent/equipment contribution; R. G. C. provided consultation and performed advanced oxidation protein products, total ketone bodies and creatinine assay validation; S. M. provided consultation, performed leptin, adiponectin, IGF-1, IL- 6 and TNF- $\alpha$ assays and provided reagent/ equipment contribution; R. W. performed leptin, adiponectin, IGF-1, IL-6 and TNF- $\alpha$ assays; S. C. performed total ketone bodies and creatinine assays; O. D. C. performed leptin, adiponectin, IGF-1, IL-6 and TNF- $\alpha$ assays; S. W. conducted the statistical analysis and provided advice; J. M. E. provided consultation, performed leptin, adiponectin, IGF-1, IL-6 and TNF- $\alpha$ assay validation and provided reagent/equipment contribution; A. H. participated in the study conception and design and manuscript preparation.

The authors declare no conflict of interest.

\section{References}

1. Loveman E, Frampton GK, Shepherd J, et al. (2011) The clinical effectiveness and cost-effectiveness of longterm weight management schemes for adults: a systematic review. Health Technol Assess 15, 1-182.

2. Henry RR, Scheaffer L \& Olefsky JM (1985) Glycemic effects of intensive caloric restriction and isocaloric refeeding in noninsulin-dependent diabetes mellitus. J Clin Endocrinol Metab 61, 917-925.

3. Wadden TA, Anderson DA \& Foster GD (1999) Two-year changes in lipids and lipoproteins associated with the maintenance of a $5 \%$ to $10 \%$ reduction in initial weight: some findings and some questions. Obes Res 7, 170-178.

4. Weinstock RS, Dai H \& Wadden TA (1998) Diet and exercise in the treatment of obesity: effects of 3 interventions on insulin resistance. Arch Intern Med 158, 2477-2483.

5. de Souza RJ, Bray GA, Carey VJ, et al. (2012) Effects of 4 weight-loss diets differing in fat, protein, and carbohydrate on fat mass, lean mass, visceral adipose tissue, and hepatic fat: results from the POUNDS LOST trial. Am J Clin Nutr 95, 614-625.

6. Tsai AG \& Wadden TA (2006) The evolution of verylow-calorie diets: an update and meta-analysis. Obesity (Silver Spring) 14, 1283-1293.

7. Anderson JW, Vichitbandra S, Qian W, et al. (1999) Long-term weight maintenance after an intensive weightloss program. J Am Coll Nutr 18, 620-627. 
8. Greenberg I, Stampfer MJ, Schwarzfuchs D, et al. (2009) Adherence and success in long-term weight loss diets: the dietary intervention randomized controlled trial (DIRECT). J Am Coll Nutr 28, 159-168.

9. Sacks FM, Bray GA, Carey VJ, et al. (2009) Comparison of weight-loss diets with different compositions of fat, protein, and carbohydrates. $N$ Engl J Med 360, 859-873.

10. Shai I, Spence JD, Schwarzfuchs D, et al. (2010) Dietary intervention to reverse carotid atherosclerosis. Circulation 121, 1200-1208.

11. Wycherley TP, Moran LJ, Clifton PM, et al. (2012) Effects of energy-restricted high-protein, low-fat compared with standard-protein, low-fat diets: a meta-analysis of randomized controlled trials. Am J Clin Nutr 96, 1281-1298.

12. Harvie M \& Howell A (2012) Energy restriction and the prevention of breast cancer. Proc Nutr Soc 71, 263-275.

13. Sogawa H \& Kubo C (2000) Influence of short-term repeated fasting on the longevity of female (NZB (NZW) F1 mice. Mech Ageing Dev 115, 61-71.

14. Cleary MP \& Grossmann ME (2011) The manner in which calories are restricted impacts mammary tumor cancer prevention. J Carcinog 10, 21.

15. Bonorden MJ, Rogozina OP, Kluczny CM, et al. (2009) Intermittent calorie restriction delays prostate tumor detection and increases survival time in TRAMP mice. Nutr Cancer 61, 265-275.

16. Susan Lanza-Jacoby, Guang Yan, Glenn Radice, et al. (2011) Abstract B4: calorie restriction prevents the development of pancreatic cancer in Kras; Pdx-1Cre mice. Cancer Prev Res 4, B4

17. Mattson MP \& Wan R (2005) Beneficial effects of intermittent fasting and caloric restriction on the cardiovascular and cerebrovascular systems. J Nutr Biochem 16, 129-137.

18. Stranahan AM \& Mattson MP (2012) Recruiting adaptive cellular stress responses for successful brain ageing. Nat Rev Neurosci 13, 209-216.

19. Stote KS, Baer DJ, Spears K, et al. (2007) A controlled trial of reduced meal frequency without caloric restriction in healthy, normal-weight, middle-aged adults. Am J Clin Nutr 85, 981-988.

20. Varady KA (2011) Intermittent versus daily calorie restriction: which diet regimen is more effective for weight loss? Obes Rev 12, e593-e601.

21. Ash S, Reeves MM, Yeo S, et al. (2003) Effect of intensive dietetic interventions on weight and glycaemic control in overweight men with Type II diabetes: a randomised trial. Int J Obes Relat Metab Disord 27, 797-802.

22. Harvie MN, Pegington M, Mattson MP, et al. (2011) The effects of intermittent or continuous energy restriction on weight loss and metabolic disease risk markers: a randomized trial in young overweight women. Int $J$ Obes (Lond) 35, 714-727.

23. Hill JO, Schlundt DG, Sbrocco T, et al. (1989) Evaluation of an alternating-calorie diet with and without exercise in the treatment of obesity. Am J Clin Nutr 50, 248-254.

24. Tyrer J, Duffy SW \& Cuzick J (2004) A breast cancer prediction model incorporating familial and personal risk factors. Stat Med 23, 1111-1130.

25. Ainsworth BE, Haskell WL, Herrmann SD, et al. (2011) Compendium of physical activities: a second update of codes and MET values. Med Sci Sports Exerc 43, 1575-1581.

26. Schofield WN (1985) Predicting basal metabolic rate, new standards and review of previous work. Hum Nutr Clin Nutr 39, Suppl. 1, 5-41.
27. Shai I, Schwarzfuchs D, Henkin Y, et al. (2008) Weight loss with a low-carbohydrate, Mediterranean, or low-fat diet. $N$ Engl J Med 359, 229-241.

28. Buckland G, Bach A \& Serra-Majem L (2008) Obesity and the Mediterranean diet: a systematic review of observational and intervention studies. Obes Rev 9, 582-593.

29. Mutrie N \& Blamey A (2004) Thinking About Becoming More Active? Glasgow: NHS Greater Glasgow Clyde.

30. Michie S, Ashford S, Sniehotta FF, et al. (2011) A refined taxonomy of behaviour change techniques to help people change their physical activity and healthy eating behaviours: the CALO-RE taxonomy. Psychol Health 26, 1479-1498.

31. Matthews DR, Hosker JP, Rudenski AS, et al. (1985) Homeostasis model assessment: insulin resistance and beta-cell function from fasting plasma glucose and insulin concentrations in man. Diabetologia 28, 412-419.

32. Friedewald WT, Levy RI \& Fredrickson DS (1972) Estimation of the concentration of low-density lipoprotein cholesterol in plasma, without use of the preparative ultracentrifuge. Clin Chem 18, 499-502.

33. Selmeci L, Seres L, Antal M, et al. (2005) Advanced oxidation protein products (AOPP) for monitoring oxidative stress in critically ill patients: a simple, fast and inexpensive automated technique. Clin Chem Lab Med 43, 294-297.

34. Grossmann ME, Ray A, Dogan S, et al. (2008) Balance of adiponectin and leptin modulates breast cancer cell growth. Cell Res 18, 1154-1156.

35. Finucane FM, Luan J, Wareham NJ, et al. (2009) Correlation of the leptin:adiponectin ratio with measures of insulin resistance in non-diabetic individuals. Diabetologia $\mathbf{5 2}$, $2345-2349$.

36. McTiernan A, Rajan KB, Tworoger SS, et al. (2003) Adiposity and sex hormones in postmenopausal breast cancer survivors. J Clin Oncol 21, 1961-1966.

37. Johnstone AM, Faber P, Gibney ER, et al. (2002) Effect of an acute fast on energy compensation and feeding behaviour in lean men and women. Int J Obes Relat Metab Disord 26, $1623-1628$.

38. Bouchard C, Tremblay A, Leblanc C, et al. (1983) A method to assess energy expenditure in children and adults. $\mathrm{Am} \mathrm{J}$ Clin Nutr 37, 461-467.

39. Mc Nair DM, Lorr M \& Droppleman LF (1971) Profile of Mood States Manual. San Diego, CA: Educational and Industrial Testing Service.

40. Wallace TM, Levy JC \& Matthews DR (2004) Use and abuse of HOMA modeling. Diabetes Care 27, 1487-1495.

41. Alberti KG, Eckel RH, Grundy SM, et al. (2009) Harmonizing the metabolic syndrome: a joint interim statement of the International Diabetes Federation Task Force on Epidemiology and Prevention; National Heart, Lung, and Blood Institute; American Heart Association; World Heart Federation; International Atherosclerosis Society; and International Association for the Study of Obesity. Circulation 120, $1640-1645$

42. Gardner CD, Kim S, Bersamin A, et al. (2010) Micronutrient quality of weight-loss diets that focus on macronutrients: results from the A TO Z study. Am J Clin Nutr 92, 304-312.

43. Schwingshackl L, Strasser B \& Hoffmann G (2011) Effects of monounsaturated fatty acids on glycaemic control in patients with abnormal glucose metabolism: a systematic review and meta-analysis. Ann Nutr Metab 58, 290-296.

44. Jebb SA, Lovegrove JA, Griffin BA, et al. (2010) Effect of changing the amount and type of fat and carbohydrate on insulin sensitivity and cardiovascular risk: the RISCK (Reading, Imperial, Surrey, Cambridge, and Kings) trial. Am J Clin Nutr 92, 748-758. 
45. Kirk E, Reeds DN, Finck BN, et al. (2009) Dietary fat and carbohydrates differentially alter insulin sensitivity during caloric restriction. Gastroenterology 136, 1552-1560.

46. Stanford J, Kaiser M, Ablah E, et al. (2012) The effect of weight loss on fasting blood sugars and hemoglobin A1c in overweight and obese diabetics and non-diabetics. J Diabetes Mellitus 2, 126-130.

47. Chalkiadaki A \& Guarente L (2012) Sirtuins mediate mammalian metabolic responses to nutrient availability. Nat Rev Endocrinol 8, 287-296.

48. Kiens B, Alsted TJ \& Jeppesen J (2011) Factors regulating fat oxidation in human skeletal muscle. Obes Rev 12, 852-858.

49. Acharya SD, Elci OU, Sereika SM, et al. (2009) Adherence to a behavioral weight loss treatment program enhances weight loss and improvements in biomarkers. Patient Prefer Adherence 3, 151-160.

50. Varady KA, Bhutani S, Church EC, et al. (2009) Short-term modified alternate-day fasting: a novel dietary strategy for weight loss and cardioprotection in obese adults. Am J Clin Nutr 90, 1138-1143.

51. Johnson JB, Summer W, Cutler RG, et al. (2007) Alternate day calorie restriction improves clinical findings and reduces markers of oxidative stress and inflammation in overweight adults with moderate asthma. Free Radic Biol Med 42, 665-674.

52. Krieger JW, Sitren HS, Daniels MJ, et al. (2006) Effects of variation in protein and carbohydrate intake on body mass and composition during energy restriction: a meta-regression 1. Am J Clin Nutr 83, 260-274.

53. Veldhorst MA, Westerterp KR, van Vught AJ, et al. (2010) Presence or absence of carbohydrates and the proportion of fat in a high-protein diet affect appetite suppression but not energy expenditure in normal-weight human subjects fed in energy balance. Br J Nutr 104, 1395-1405.

54. Macdiarmid J \& Blundell J (1998) Assessing dietary intake: Who, what and why of under-reporting. Nutr Res Rev 11, 231-253.

55. Dansinger ML, Gleason JA, Griffith JL, et al. (2005) Comparison of the Atkins, Ornish, Weight Watchers, and Zone diets for weight loss and heart disease risk reduction: a randomized trial. JAMA 293, 43-53.
56. Summerbell CD, Watts C, Higgins JP, et al. (1998) Randomised controlled trial of novel, simple, and well supervised weight reducing diets in outpatients. BMJ 317, 1487-1489.

57. Johnstone AM, Horgan GW, Murison SD, et al. (2008) Effects of a high-protein ketogenic diet on hunger, appetite, and weight loss in obese men feeding ad libitum. Am J Clin Nutr 87, 44-55.

58. Leichtle AB, Helmschrodt C, Ceglarek U, et al. (2011) Effects of a 2-y dietary weight-loss intervention on cholesterol metabolism in moderately obese men. Am J Clin Nutr 94 , $1189-1195$.

59. Reid IR \& Bolland MJ (2012) Calcium supplements: bad for the heart? Heart 98, 895-896.

60. Brinkworth GD, Noakes M, Buckley JD, et al. (2009) Longterm effects of a very-low-carbohydrate weight loss diet compared with an isocaloric low-fat diet after $12 \mathrm{mo}$. Am J Clin Nutr 90, 23-32.

61. Alexander DD, Weed DL, Cushing CA, et al. (2011) Metaanalysis of prospective studies of red meat consumption and colorectal cancer. Eur J Cancer Prev 20, 293-307.

62. Johnstone AM (2012) Safety and efficacy of high-protein diets for weight loss. Proc Nutr Soc 71, 339-349.

63. Tonolo G, Ciccarese M, Brizzi P, et al. (1995) Cyclical variation of plasma lipids, apolipoproteins, and lipoprotein(a) during menstrual cycle of normal women. Am J Physiol 269, E1101-E1105.

64. Anttila L, Koskinen P, Irjala K, et al. (1991) Reference intervals for serum sex steroids and gonadotropins in regularly menstruating women. Acta Obstet Gynecol Scand 70, 475-481.

65. Thomson R, Brinkworth GD, Buckley JD, et al. (2007) Good agreement between bioelectrical impedance and dualenergy X-ray absorptiometry for estimating changes in body composition during weight loss in overweight young women. Clin Nutr 26, 771-777.

66. Jebb SA, Siervo M, Murgatroyd PR, et al. (2007) Validity of the leg-to-leg bioimpedance to estimate changes in body fat during weight loss and regain in overweight women: a comparison with multi-compartment models. Int $J$ Obes (Lond) 31, 756-762. 